\title{
On-line Appendix for Money and Asset Liquidity in Frictional Capital Markets
}

\author{
Wei Cui and Sören Radde
}

\section{Equilibrium Conditions}

Assuming both private claims and money co-exist. Define $L_{t} \equiv B_{t} / P_{t-1}$ and inflation $\Pi_{t} \equiv$ $P_{t} / P_{t-1}$. We also substitute $S_{t}$ by $S_{t}=K_{t}$. Then, we solve $\left(K_{t+1}, L_{t+1}, C_{t}, I_{t}, r_{t}, \Pi_{t}, q_{t}, q_{t}^{i}\right.$, $\left.q_{t}^{n}, \rho_{t}, \phi_{t}, f_{t}\right)$, given $\left(K_{t}, L_{t}, A_{t}\right)$ with the following equilibrium conditions collected from the main text:

$$
\begin{aligned}
& r_{t}=A_{t} \alpha\left(K_{t} / \bar{N}\right)^{\alpha-1} \\
& 1=\mathbb{E}_{t}\left[\frac{\beta u^{\prime}\left(C_{t+1}\right)}{u^{\prime}\left(C_{t}\right)} \frac{\chi \rho_{t+1}+1-\chi}{\Pi_{t+1}}\right] \\
& 1=\mathbb{E}_{t}\left[\frac{\beta u^{\prime}\left(C_{t+1}\right)}{u^{\prime}\left(C_{t}\right)} \frac{\left[\left(\chi \rho_{t+1}+1-\chi\right) r_{t+1}+\left[\chi \rho_{t+1}+(1-\chi) q^{n}\right](1-\delta)\right]}{q_{t}^{n}}\right] \\
& I_{t}=\frac{\left[r_{t}+(1-\delta) \phi_{t} q_{t}^{i}\right] \chi K_{t}+\chi L_{t} / \Pi_{t}}{1-\phi_{t} q_{t}^{i}} \\
& q^{i}=1+\frac{\kappa \eta}{1-\eta} \frac{(1-\phi)}{f} \\
& q_{t}^{n}-q_{t}^{i}=\kappa\left[\phi^{-1}+f^{-1}\right] \\
& f_{t}=\xi^{\frac{1}{1-\eta}} \phi_{t}^{\frac{\eta}{\eta-1}} \\
& \rho_{t}=\frac{q_{t}^{n}}{q_{t}^{r}}=\frac{\left(1-\phi_{t}\right) q_{t}^{n}}{1-\phi_{t} q_{t}^{i}} \\
& C_{t}+L_{t+1}+q_{t}^{n} K_{t+1}=\left[\frac{(1-\alpha)}{\alpha}+\chi \rho_{t}+1-\chi\right] r_{t} K_{t} \\
& +\left[\chi \rho_{t}+(1-\chi) q_{t}^{n}\right](1-\delta) K_{t}+\left[\chi \rho_{t}+1-\chi\right] \frac{L_{t}}{\Pi_{t}} \\
& K_{t+1}=(1-\delta) K_{t}+I_{t} \\
& L_{t+1}=\frac{L_{t}}{\Pi_{t}}
\end{aligned}
$$


where we have used the fact that $w_{t} N_{t}=\frac{(1-\alpha) r_{t} K_{t}}{\alpha}$ in the household budget constraint (9). The co-existence of money and private claims is equivalent to that (2), (3), $L_{t} \geq 0$, and $\phi_{t}>0$ all satisfy.

In steady state, $\Pi=1$ and we know from the Euler equation for money (2) that

$$
\rho=\rho^{*} \equiv 1+\frac{\beta^{-1}-1}{\chi}
$$

Using the definition of $\rho$ in (8), we know that

$$
(1-\phi) q^{n}-\rho\left(1-\phi q^{i}\right)=0
$$

where

$$
\begin{aligned}
q^{i} & =1+\frac{\kappa \eta \xi^{\frac{1}{\eta-1}}}{1-\eta}(1-\phi) \phi^{\frac{\eta}{1-\eta}} \\
q^{n} & =q^{i}+\kappa\left[\phi^{-1}+\xi^{\frac{1}{\eta-1}} \phi^{\frac{\eta}{1-\eta}}\right]
\end{aligned}
$$

Then, we know that $\phi$ is a solution to equation (14), which only depends on parameters $\beta$, $\chi, \kappa$, and $\eta$. Once we know $\phi$, we can solve $f=\xi^{\frac{1}{1-\eta}} \phi^{\frac{\eta}{\eta-1}}, q^{n}$, and $q^{i}$.

So far, we obtain all relevant variables in the financial market. Now, we solve for real variables. Using (1), we know that the marginal product of capital and the level of capital stock are

$$
\begin{gathered}
r=\delta q^{n}+\left(\beta^{-1}-1+\chi\right)\left(q^{n}-1\right) \\
K=\left(\frac{r}{A \alpha}\right)^{\frac{1}{\alpha-1}}
\end{gathered}
$$

We then use the investment equation (4) and the relationship $I=\delta K$ from (11) to derive the real value of liquidity

$$
L=\left[\delta\left(1-\phi q^{i}\right) / \chi-\left(r+(1-\delta) \phi q^{i}\right)\right] K
$$

Our assumption is correct if $L \geq 0$. Finally, consumption $C$ can be solved directly from the household's budget constraint (9):

$$
C=\left[\frac{(1-\alpha) r}{\alpha}+q^{n}\left(\beta^{-1}-1\right)\right] K+\chi(\rho-1) L
$$

\section{Proofs}

We follow the notation in the main text. Since we can omit sub-market superscript $m$, we can just write $\phi, f, q^{n}, q^{i}$, and $q$ (instead of $\phi^{m}, f^{m}, q^{n, m}, q^{i, m}$, and $q^{m}$ )

Proof to optimal $\left(\phi, q^{i}\right)$ pairs. Since $\frac{\kappa}{f}=q-q^{i}$, then $1-\phi q^{i}=\frac{\kappa \phi}{f}+1-\phi q$ and

$$
q^{r}=\frac{1-\phi q^{i}}{1-\phi}=\frac{\frac{\kappa \phi}{f}+1-\phi q}{1-\phi}
$$


To minimize $q^{r}$, we have the first-order condition with respect to $\phi$ as

$$
\frac{d q^{r}(\phi)}{d \phi}=\frac{(1-\phi)\left[\frac{\kappa}{(1-\eta) f}-q\right]+\left[\frac{\kappa \phi}{f}+1-\phi q\right]}{(1-\phi)^{2}}=0
$$

where we have used the fact that $f=\xi^{\frac{1}{1-\eta}} \phi^{\frac{\eta}{\eta-1}}$. Rearranging, we have

$$
q=1+\kappa\left[\frac{1-\phi}{(1-\eta) f}+\frac{\phi}{f}\right]
$$

which implies that

$$
q^{i}=q-\frac{\kappa}{f}=1+\frac{\kappa \eta(1-\phi)}{(1-\eta) f}
$$

as shown in the main text. Given $q^{n}, \phi$ can be backed out from $\frac{\kappa}{f}=q^{n}-q^{i}$ and $f=\xi^{\frac{1}{1-\eta}} \phi^{\frac{\eta}{\eta-1}}$.

Finally, we check the second-order condition to ensure minimization. Using (21), we can express

$$
\frac{d q^{r}(\phi)}{d \phi}=\frac{\kappa}{(1-\eta)} \frac{1}{(1-\phi) f}+\frac{1-q}{(1-\phi)^{2}}+\frac{\kappa \phi}{f(1-\phi)^{2}}
$$

Then, we use again $f=\xi^{\frac{1}{1-\eta}} \phi^{\frac{\eta}{\eta-1}}$ to derive

$$
\frac{d^{2} q^{r}(\phi)}{d \phi^{2}}=\frac{\kappa}{(1-\eta)} \frac{\frac{\eta}{1-\eta} \frac{1-\phi}{\phi f}+\frac{1}{f}}{(1-\phi)^{2}}+\frac{2(1-q)}{(1-\phi)^{3}}+\kappa \frac{\frac{(1-\phi)^{2}}{(1-\eta) f}+\frac{2 \phi}{f}(1-\phi)}{(1-\phi)^{4}}
$$

Plug in the expression of $q$ from (22):

$$
\begin{aligned}
\frac{d^{2} q^{r}(\phi)}{d \phi^{2}} & =\frac{\kappa}{(1-\eta)} \frac{\frac{\eta}{1-\eta} \frac{1-\phi}{\phi f}+\frac{1}{f}}{(1-\phi)^{2}}-\kappa \frac{\frac{2 \phi}{f}+\frac{2(1-\phi)}{(1-\eta) f}}{(1-\phi)^{3}}+\kappa \frac{\frac{(1-\phi)^{2}}{(1-\eta) f}+\frac{2 \phi}{f}(1-\phi)}{(1-\phi)^{4}} \\
& =\frac{\kappa}{(1-\eta)} \frac{\frac{\eta}{1-\eta} \frac{1-\phi}{\phi f}+\frac{1}{f}}{(1-\phi)^{2}}-\kappa \frac{\frac{1}{(1-\eta) f}}{(1-\phi)^{2}} \\
& =\frac{\kappa \eta}{(1-\eta)^{2}} \frac{1}{\phi(1-\phi) f}>0
\end{aligned}
$$

The first-order condition is thus verified to be sufficient and necessary.

Proof to Proposition 1: If private claims and money co-exist, we already know that $\rho=\rho^{*}=1+\frac{\beta^{-1}-1}{\chi}>1$ (from the Euler equation for money $(2)$ ). The steady state liquidity premium can be written as

$$
\begin{aligned}
\Delta^{L P} & =\chi r^{n i}+\frac{(1-\chi) r^{n n}}{\rho}+(1-\chi)\left(1-\rho^{-1}\right) r^{n n}-1 \\
& =\rho^{-1} \beta^{-1}-1+(1-\chi)\left(1-\rho^{-1}\right) r^{n n} \\
& =\rho^{-1}(\chi \rho+1-\chi)-1+(1-\chi)\left(1-\rho^{-1}\right) r^{n n} \\
& =(1-\chi)\left(1-\rho^{-1}\right)\left(r^{n n}-1\right) \\
& =(1-\chi)\left(1-\rho^{-1}\right)\left(r / q^{n}-\delta\right)
\end{aligned}
$$


where we use (3) in the second equality. Further, using (17) obtained from the Euler equation for private claims (3), we have

$$
\begin{aligned}
\frac{r}{q^{n}} & =\delta+\left[\beta^{-1}-1+\chi\right]\left[1-\frac{1}{q^{n}}\right] \\
& =\delta+\chi \rho\left[1-\frac{1}{q^{n}}\right]>\delta
\end{aligned}
$$

as $q^{n}>q^{i}>1$ when $\kappa>0$. Then, the liquidity premium

$$
\begin{aligned}
\Delta^{L P} & =\left(1-\rho^{-1}\right)\left(r / q^{n}-\delta\right)(1-\chi) \\
& =(\rho-1) \chi(1-\chi)\left[1-\frac{1}{q^{n}}\right]>0 .
\end{aligned}
$$

\section{A Numerical Example}

We calibrate the model by setting $\beta=0.99, \delta=0.03$, and $\alpha=0.33$ as in a standard calibration for a quarterly model. We set $\chi=0.056$ as discussed in the main text. In the financial market, $\eta=1 / 2, \xi=0.20$, and $\kappa=0.01$.

With $\eta=\frac{1}{2}$, we can substitute $q^{i}$ and $q^{n}$ to have a quartic equation. To see this, by using the relationship between $q^{i}$ and $q^{n}$, we have

$$
q^{i}\left[\left(\rho^{*}-1\right) \phi+1\right] \phi=\left[\frac{\kappa}{\xi^{2}} \phi^{3}-\frac{\kappa}{\xi^{2}} \phi^{2}+\left(\rho^{*}+\kappa\right) \phi-\kappa\right]
$$

Using $q^{i}=1+\frac{\kappa}{\xi^{2}} \phi-\frac{\kappa}{\xi^{2}} \phi^{2}$, we obtain

$$
a \phi^{4}+b \phi^{3}+c \phi^{2}+d \phi+e=0
$$

where the coefficients are

$$
\begin{gathered}
a=\frac{\kappa\left(\rho^{*}-1\right)}{\xi^{2}}, \quad b=\frac{\kappa\left(3-\rho^{*}\right)}{\xi^{2}} \\
c=-\left(\frac{2 \kappa}{\xi^{2}}+\rho^{*}-1\right), \quad d=\rho^{*}-1+\kappa, \quad e=-\kappa
\end{gathered}
$$

Therefore, $\phi$ solves the quartic equation (24).

(13) implies that $\rho^{*}=1.1804$. Solving $(24)$ in $(0,1)$, we obtain two roots:

$$
\phi_{1}=0.0685 \text { and } \phi_{2}=0.2828
$$

Then, we can compute $q^{i}$ and $q^{n}$ from (15) and (16). With the knowledge of $q^{n}$, we can compute $r, K, L, C$ from (17), (18), (19), and (20). Finally, output $Y=r K / \alpha$ because of the Cobb-Douglas production function. 